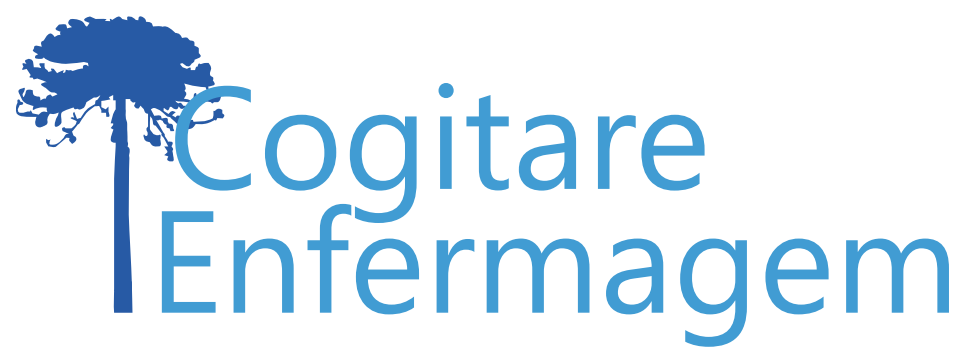

\title{
UTILIZAÇÃO DA SIMULAÇÃO CLÍNICA NO ENSINO DE ENFERMAGEM NO BRASIL: CONDIÇÕES DIANTE DA PANDEMIA DE COVID-19
}

Raphael Raniere de Oliveira Costa ${ }^{1}$ (i) Rodrigo Guimarães dos Santos Almeida² (1) Alessandra Mazzo ${ }^{3}$ (i)

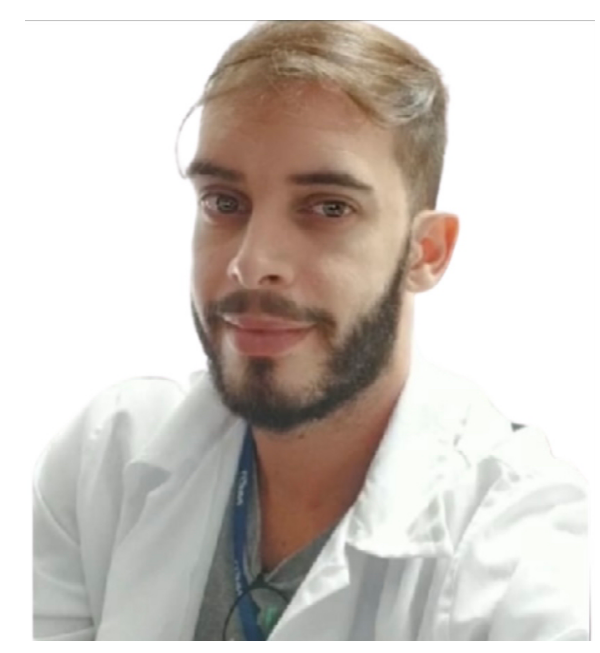

Raphael Raniere de Oliveira Costa

DESCRITORES: Enfermagem; Simulação; Pandemias.

O editorial "A simulação soluciona o problema da aprendizagem clínica no ensino de enfermagem em tempos da pandemia provocada pela covid-19?"(1) nos leva à reflexão sobre a utilização da simulação clínica como estratégia pedagógica para o ensino da prática em enfermagem, e até mesmo para o ensino das demais profissões da área da saúde.

São sabidas as inúmeras contribuições que a simulação clínica proporciona para a formação em saúde, como a autoconfiança, a melhoria do desempenho cognitivo, a autoeficácia, o desenvolvimento de competências e habilidades técnicas e não técnicas, entre outras ${ }^{(2-4)}$. Há avanços consideráveis, sobretudo nos últimos 10 anos, nas formas de se fazer a simulação e nos seus recursos/tecnologias. Entretanto, no contexto brasileiro, ainda há escolas de enfermagem que dispõem de poucos recursos ou até mesmo onde inexistem laboratórios de habilidades estruturados. Muitas dessas instituições não vislumbram, a curto e médio prazos, a inserção curricular da simulação clínica enquanto método de ensino e aprendizagem. Nesses contextos, muitos formandos desenvolvem suas habilidades diretamente nos campos de prática. 
Com o advento da pandemia causada pelo vírus Sars-CoV-2, a escassez de recursos nas instituições de ensino, associada às mudanças dos campos de prática, tem prejudicado o processo de ensino e formação, principalmente a continuidade das práticas em laboratório e a inserção dos estudantes nos cenários de práticas. Essas dificuldades giram em torno da implementação e da garantia do cumprimento dos protocolos de biossegurança; das limitações de estrutura física adequada; dos recursos materiais utilizados para o treino de habilidades; do quantitativo de simuladores; das dificuldades/limitações financeiras para a aquisição de materiais, como softwares de realidade virtual; da contratação de assessoria especializada, entre outros fatores. No entanto, a principal dificuldade decorre do conhecimento restrito dos formadores sobre a estratégia de simulação clínica e de telessimulação.

Nessa perspectiva, no contexto brasileiro, algumas universidades e associações da área de simulação clínica têm ofertado encontros remotos para a troca de experiências exitosas, com a finalidade de favorecer o amplo uso de experiências clínicas simuladas presenciais em ambientes seguros e também de experiências remotas. Ao considerar as diferenças geográficas do Brasil, bem como as diversas realidades dos cursos de graduação em enfermagem nas distintas regiões do país, é indispensável que se promovam estratégias de integração e de fortalecimento da simulação clínica enquanto método potencial e viável, em tempos de pandemia, não só para o trabalho remoto, mas também para o presencial em ambientes biologicamente seguros.

Portanto, é importante que se compartilhem ideias exitosas, criativas e inovadoras. Além disso, é importante destacar que não podemos incorporar - sem discussão alguma recomendações sobre tempo de prática, tempo de laboratório, permissões ou restrições. É importante, antes dessas incorporações, vislumbrar as dificuldades e as necessidades locais e regionais, assim como ter em conta o trabalho criterioso de pesquisadores que levam em consideração as características da nossa população e as particularidades, potencialidades e fragilidades da simulação clínica.

Todavia, salienta-se que a utilização de simulação clínica no ensino não deve, em momento algum, substituir as práticas clínicas nos serviços de saúde e a interação com os pacientes ${ }^{(5-6)}$. A simulação é uma etapa do processo de formação, que prepara melhor o aprendiz para atender o paciente de forma mais segura e com melhor qualidade. Porém, a utilização da estratégia e de suas ramificações, como a telessimulação, são possibilidades de complementar os momentos de formação em saúde em contextos como os atuais, em que os serviços e as instituições possuem limitações de espaços de práticas sem uma previsão de retorno seguro, haja visto as condições de vida que a pandemia de Covid-19 impôs a toda a sociedade.

\section{REFERÊNCIAS}

1. Marques P. A simulação soluciona o problema da aprendizagem clínica no ensino de enfermagem em tempos de pandemia provocada pela COVID-19? Cogit. Enferm. [Internet]. 2021 [acesso em 20 maio 2021]: 26:e81207. Disponível em: http://dx.doi.org/10.5380/ce.v26i0.78603.

2. Costa RRO, Medeiros SM, Martins JCA, Coutinho VRD, Araújo MS. Effectiveness of simulation in teaching immunization in nursing: a randomized clinical trial. Rev Lat Am Enfermagem. [Internet]. 2020 [acesso em 22 maio 2021]: 19(28):e3305. Disponível em: https://doi.org/10.1590/1518-8345.3147.3305.

3. Costa RRO, Medeiros SM, Coutinho VRD, Mazzo A, Araújo MS. Satisfaction and self-confidence in the learning of nursing students: Randomized clinical trial. Esc. Anna Nery. [Internet]. 2020 [acesso em 22 maio 2021]: 24 (1): e20190094. Disponível em: https://doi.org/10.1590/2177-9465-EAN-2019-0094.

4. Weiler DT, Gibson AL, Saleem JJ. The effect of role assignment in high fidelity patient simulation on 
nursing students: An experimental research study. Nurse Educ Today. [Internet]. 2018 [acesso em 22 maio 2021]: 63:29-34. Disponível em: https://pubmed.ncbi.n/m.nih.gov/29407257/.

5. Costa RRO, Medeiros SM, Martins JCA, Coutinho VRD. A simulação no ensino de enfermagem: reflexões e justificativas à luz da bioética e dos direitos humanos. Acta Bioeth. [Internet]. 2018 jun; [acesso em 22 maio 2021]; 24(1):31-8. Disponível em: http://dx.doi.org/10.4067/S1726-569X2018000100031.

6. Jeffries P. The Good News-Simulations Work, So Now What? J Nurs Educ. [Internet]. 2015 [acesso em 22 mai 2021]; 54(11): 603-60. Disponível em: https://doi.org/10.3928/01484834-20151016-10.

\section{COMO REFERENCIAR ESTE ARTIGO:}

Costa RR de O, Almeida RG dos S, Mazzo. Utilização da simulação clínica no ensino de enfermagem no brasil: condições diante da pandemia de covid-19. Cogit. Enferm. [Internet]. 2021 [acesso em "colocar data de acesso, dia, mês abreviado e ano"]; 26. Disponível em: http://dx.doi.org/10.5380/ce.v26i0.81207.

Recebido em: 26/05/2021

Aprovado em: 20/06/2021

Editora associada: Luciana Puchalski Kalinke

Autor Correspondente:

Raphael Raniere de Oliveira Costa

Universidade Federal do Rio Grande do Norte - Caicó, RN, Brasil

E-mail: raphael.costa@ufrn.br

\section{Contribuição dos autores:}

Contribuições substanciais para a concepção ou desenho do estudo; ou a aquisição, análise ou interpretação de dados do estudo - RROC, RGSA, AM

Elaboração e revisão crítica do conteúdo intelectual do estudo - RROC, RGSA, AM

Aprovação da versão final do estudo a ser publicado - RROC, RGSA, AM

Responsável por todos os aspectos do estudo, assegurando as questões de precisão ou integridade de qualquer parte do estudo - RROC, RGSA, AM

Copyright ( 2021 Este é um artigo em acesso aberto distribuído nos termos da Licença Creative Commons Atribuição, que permite o uso irrestrito, a distribuição e reprodução em qualquer meio desde que o artigo original seja devidamente citado. 